\title{
A Young Woman with Ischemic Stroke: Should We Pay More Attention to Varicella Zoster Infection?
}

\author{
Cláudia Borbinha ${ }^{a}$ João Pedro Marto ${ }^{a}$ Sofia Calado ${ }^{a, b}$ \\ Miguel Viana-Baptista ${ }^{a, b}$ \\ ${ }^{a}$ Neurology Department, Hospital Egas Moniz - Centro Hospitalar de Lisboa \\ Ocidental, Lisbon, Portugal; ${ }^{b}$ CEDOC, Nova Medical School/Faculdade de Ciências \\ Médicas, Universidade Nova de Lisboa, Lisbon, Portugal
}

\section{Keywords}

Varicella zoster virus · Vasculopathy · Ischemic stroke · Hemorrhagic stroke · Young adults . Anticoagulation

\begin{abstract}
Ischemic and hemorrhagic stroke are recognized complications of Varicella zoster virus (VZV) infections, although uncommon and poorly documented. The authors report the case of a 31-year-old woman admitted with acute ischemic stroke of the right posterior cerebral artery and a history of a thoracic rash 1 month before. Aspirin and simvastatin were prescribed, but the patient suffered a stepwise deterioration the following days, with new areas of infarction on brain imaging. Despite no evidence of cardiac or large vessel embolic sources, anticoagulation was started empirically 6 days after stroke onset. One week later, symptomatic hemorrhagic transformation occurred. The diagnosis of VZV vasculopathy was then considered, and treatment with acyclovir and prednisolone was started with no further vascular events. Cerebrospinal fluid analysis and digital subtraction angiography findings corroborated the diagnosis. The patient was discharged to the rehabilitation center with a modified Rankin scale (mRS) score of 4. On the 6-month follow-up, she presented only a slight disability (mRS score 2). In conclusion, VZV vasculopathy needs to be considered in young adults with stroke. A
\end{abstract}


high index of suspicion and early treatment seem to be important to minimize morbidity and mortality. Anticoagulation should probably be avoided in stroke associated with VZV vasculopathy.

\section{Introduction}

Varicella zoster virus (VZV) is a neurotropic DNA alpha herpesvirus that infects more than $95 \%$ of the world population. Usually, varicella results from primary infection. The virus then becomes latent in ganglion neurons along the entire neuroaxis, and after reactivation, VZV moves transaxonally to the skin resulting in zoster [1]. Less often, VZV reactivation leads to neurologic complications more common in immunocompromised patients, such as VZV vasculopathy [2]. The typical presentation in adults is ophthalmic zoster followed by acute contralateral hemiplegia [3]. Vasculitis has, however, been reported in patients with dermatomal herpes zoster infection without trigeminal nerve involvement. The average time from rash to neurologic symptoms and signs is 4.1 months, but sporadically the patient can present with rash and stroke simultaneously. Nonetheless, approximately one third of patients have no history of rash [2]. In recent years, the number of recognized VZV vasculopathies has grown [1], but the diagnosis is not easy because, frequently, clinical presentation and imaging features are not specific of this entity. Antiviral and antithrombotic treatment should probably be considered in stroke associated with VZV vasculopathy, but data on which to base therapeutic decisions are lacking.

\section{Case Report}

A 31-year-old right-handed female was admitted to the emergency department because of headache associated with visual disturbance. She had no previous history of headache and no known vascular risk factors but she used an oral contraceptive pill. On admission, she reported a 9-hour progressive pulsatile headache, with nausea and photophobia, followed by a sudden left visual field defect. Neurological examination documented left homonymous hemianopia. A head computed tomography (CT) scan was judged to be normal (fig. 1a). She was treated with paracetamol and metoclopramide with subjective improvement followed by hospital discharge. The next day, the patient was observed at an outpatient consultation and subsequently admitted in the neurology ward because the headache had worsened and she felt weakness and numbness of the left limbs. She also recalled a history of thoracic rash 1 month before and she reported having had varicella at the age of 7 years. Neurological examination at that time revealed slight drowsiness, mild left hemiparesis, left hemihypesthesia and homolateral homonymous hemianopia. A new CT scan showed an ischemic infarct on the territory of the right posterior cerebral artery (fig. $1 \mathrm{~b}$ ) and contrast administration excluded dural sinus venous thrombosis. Cervical and transcranial ultrasound did not disclose significant abnormalities, namely vasospasm. She was started on acetylsalicylic acid $150 \mathrm{mg}$ and simvastatin $20 \mathrm{mg}$ i.d.

Two days after admission, the neurological picture worsened with acute onset of left central facial palsy, visual and tactile inattention and worsening of the left motor deficit. A new CT scan showed enlargement of the ischemic lesion with involvement of the right thalamus (fig. 1c) and new areas of infarction involving different arterial territories, further depicted on MRI (fig. 2a, b). MR angiography showed an occlusion of the right posterior cere- 
bral artery $5 \mathrm{~mm}$ after its origin (fig. 2c). Transthoracic and transesophageal echocardiography and Holter ECG were within the norms, as were the of laboratory blood tests (including HIV antibodies). Clinical deterioration continued with further motor compromise, and although no cardiac or large vessel embolic source had been documented, facing neurological worsening, acetylsalicylic acid was substituted by low molecular weight heparin (60 $\mathrm{mg}$ subcutaneously twice a day) 6 days after admission. The patient remained stable for a week, but then a new episode occurred, with headache and slight drowsiness associated with hemorrhagic transformation involving the thalamus but sparing the cortical territory of the posterior cerebral artery (fig. 3a). Anticoagulation was stopped immediately.

Because of the medical history of recent VZV reactivation, it was then hypothesized that the stroke could be secondary to VZV vasculopathy. However, at that time, evidence of increased intracranial pressure contraindicated the lumbar puncture. Acyclovir and prednisolone were started empirically (acyclovir $750 \mathrm{mg}$ three times daily, and prednisolone $60 \mathrm{mg}$ once a day for 21 days), with slow but steady improvement. Digital subtraction angiography (DSA) showed narrowing at the origin of the right posterior cerebral artery and demonstrated an irregular lumen of the posterior segment of the pericallosal artery with beading-like pattern appearance (fig. 3b). She underwent a lumbar puncture 11 days after initiation of therapy (27 days after admission). Cerebrospinal fluid (CSF) protein content was slightly increased ( $48 \mathrm{mg} / \mathrm{dl}$ ), glucose $59 \mathrm{mg} / \mathrm{dl}$, and a discrete mononuclear pleocytosis was detected ( 6 cells). VZV-DNA was not detected by polymerase chain reaction, but there was an increased CSF/serum ratio of VZV IgG (3.3), confirming intrathecal production of anti-VZV antibodies.

Motor function improved, but the patient was discharged with persistent left homonymous hemianopia and spatial inattention, unable to walk without assistance [modified Rankin scale (mRS) score 4]. After antiviral therapy, she restarted acetylsalicylic acid $150 \mathrm{mg}$ once a day. On the 6-month follow-up, only a mild to moderate paresis of the left limbs was present. Hemianopia also, improved but an incomplete deficit of the left visual field persisted. The patient was able to walk unassisted and look after her own affairs (mRS score 2).

\section{Discussion}

This case study raised several important clinical issues. One of them was the difficulty of diagnosis with prompt treatment. Recognition of VZV vasculopathy was not straightforward because this is an uncommon entity and strokes of other etiologies may present with a similar neurologic picture and imaging abnormalities. On the other hand, the classic clinical presentation of VZV vasculopathy is acute contralateral hemiplegia after ophthalmic zoster. Finally, VZV-induced central nervous system (CNS) disease seems to be rare in immunocompetent patients [4].

Despite no previous history of headache and sudden presentation of the visual deficit, migrainous infarction could be considered in our patient on account of presentation with pulsatile progressive headache in a young female and the known predilection of this complication for the posterior circulation [5]. Cryptogenic stroke, which may be responsible for as much as $50 \%$ of strokes in young adults $[6,7]$, was also considered in the absence of findings that supported cardiac embolism, atherosclerosis, arterial dissection, inflammatory or druginduced etiologies.

Although there was no evidence to support the decision, anticoagulation was considered because of the stepwise deterioration of the neurological status under aspirin, with new 


\section{Case Reports in Neurology}

Borbinha et al.: A Young Woman with Ischemic Stroke: Should We Pay More Attention to Varicella Zoster Infection?

ischemic areas in different territories, and a MR angiography not indicative of vasculitis. Looking backwards, anticoagulation may not have been the most suitable therapeutic choice and should probably be avoided with in-patients with stroke and recent VZV infection, as hemorrhagic transformation occurred probably in the context of vessel wall damage but may also have been triggered by anticoagulation [1].

A stroke syndrome can develop after primary or secondary VZV infection. The biological mechanisms are probably multifactorial: inflammation associated with systemic infection may lead to endothelial dysfunction [3]; in addition, the VZV virus spreads along nerve fibers and directly involves the vessels [1]. Vasculitis has been described in patients with dermatomal herpes zoster infection without trigeminal nerve involvement, as in our case [8]. The vasculopathy typically involves the anterior circulation, but VZV vasculitis involvement of the vertebrobasilar system has also been reported. These studies suggest that VZV may enter the CNS not only from trigeminal ganglia but also from other pathways [9].

As suggested in previous reports, this case highlights the usefulness of DSA and CSF/serum ratio of VZV IgG for the diagnosis of VZV vasculopathy [2]. VZV DNA was not present in CSF, but several studies with serial analysis revealed its presence only during the first 2 weeks of disease, while anti-VZV IgG antibodies become detectable during the second week after infection [2]. The diagnostic value of detecting anti-VZV IgG antibody in CSF is greater than that of detecting VZV DNA [1]. The multifocal nature of VZV vasculopathy makes biopsy a test with low sensitivity and high morbidity [4]. Considering the difficulties in diagnosis, González-Suárez et al. [4] proposed diagnostic criteria for CNS VZV vasculopathy. In our case, when the diagnosis of VZV vasculopathy was considered, a lumbar puncture was contraindicated due to increased intracranial pressure; nevertheless, we began treatment with intravenous acyclovir and steroids. Although there are no controlled trials to access the optimal treatment schemes, we continued treatment during 21 days. Duration of antiviral treatment as well as acyclovir dosage and benefit of steroids are issues that deserve further research [10].

In conclusion, our case underlines that vasculopathy and stroke related to VZV infection should be borne in mind when dealing with stroke in young adults. In fact, available data suggest that this complication of a frequent infection may be more common than usually reported, not only after herpes zoster or varicella but also in cases of a stroke of unknown origin $[1,2]$. DSA seems to be important to the diagnosis of VZV vasculopathy, which is confirmed by intrathecal synthesis of anti-VZV IgG antibody. Prompt treatment with empiric intravenous acyclovir and oral prednisone should be started. Anticoagulation should probably be avoided in stroke associated with VZV vasculopathy.

\section{Statement of Ethics}

The authors have no ethical conflicts to declare.

\section{Disclosure Statement}

The authors declare that there are no conflicts of interest and that no funding was received for this report. 
Borbinha et al.: A Young Woman with Ischemic Stroke: Should We Pay More Attention to Varicella Zoster Infection?

\section{References}

1 Nagel MA, Gilden D: The relationship between herpes zoster and stroke: Curr Neurol Neurosci Rep 2015;15:16

-2 Nagel MA, Cohrs RJ, Mahalingam R, Wellish MC, Forghani B, Schiller A, Safdieh JE, Kamenkovich E, Ostrow LW, Levy M, Greenberg B, Russman AN, Katzan I, Gardner CJ, Häusler M, Nau R, Saraya T, Wada H, Goto H, de Martino M, Ueno M, Brown WD, Terborg C, Gilden DH: The varicella zoster virus vasculopathies: clinical, CSF, imaging, and virologic features. Neurology 2008;70:853.

-3 Kleinschmidt-Demasters BK, Gilden DH: Varicella-zoster virus infections of the nervous system: clinical and pathologic correlates. Arch Pathol Lab Med 2001;125:770-780.

4 González-Suárez I, Fuentes-Gimeno B, Ruiz-Ares G, Martínez-Sánchez P, Diez-Tejedor E: Varicellazoster virus vasculopathy. A review description of a new case with multifocal brain hemorrhage. J Neurol Sci 2014;338:34-38.

-5 Wolf ME, Szabo K, Griebe M, Förster A, Gass A, Hennerici MG, Kern R: Clinical and MRI characteristics of acute migrainous infarction. Neurology 2011;76:1911-1917.

-6 Kittner SJ, Stern BJ, Wozniak M, Buchholzet DW, Earley CJ, Feeser BR, Johnson CJ, Macko RF, McCarter RJ, Price TR, Sherwin R, Sloan MA, Wityk RJ: Cerebral infarction in young adults. Neurology 1998;50:890-894.

7 Amarenco P: Cryptogenic stroke, aortic arch atheroma, patent foramen ovale, and the risk of stroke. Cerebrovasc Dis 2005;20(suppl 2):68-74.

-8 Gilden DH, Lipton HL, Wolf JS, Akenbrandt W, Smith JE, Mahalingam R, Forghani B: Two patients with unusual forms of varicella-zoster virus vasculopathy. N Engl J Med 2002;347:1500-1503.

9 Matsuo K, Uozumi Y, Miyamoto H, Tatsumi S, Kohmura E: Varicella-zoster vasculitis presenting with cerebellar hemorrhage. J Stroke Cerebrovasc Dis 2015;24:e153-e155.

$\checkmark 10$ Gilden D, Cohrs RJ, Mahalingam R, Nagel MA: Varicella Zoster virus vasculopathies: diverse clinical manifestations, laboratory features, pathogenesis, and treatment. Lancet Neurol 2009;8:731.
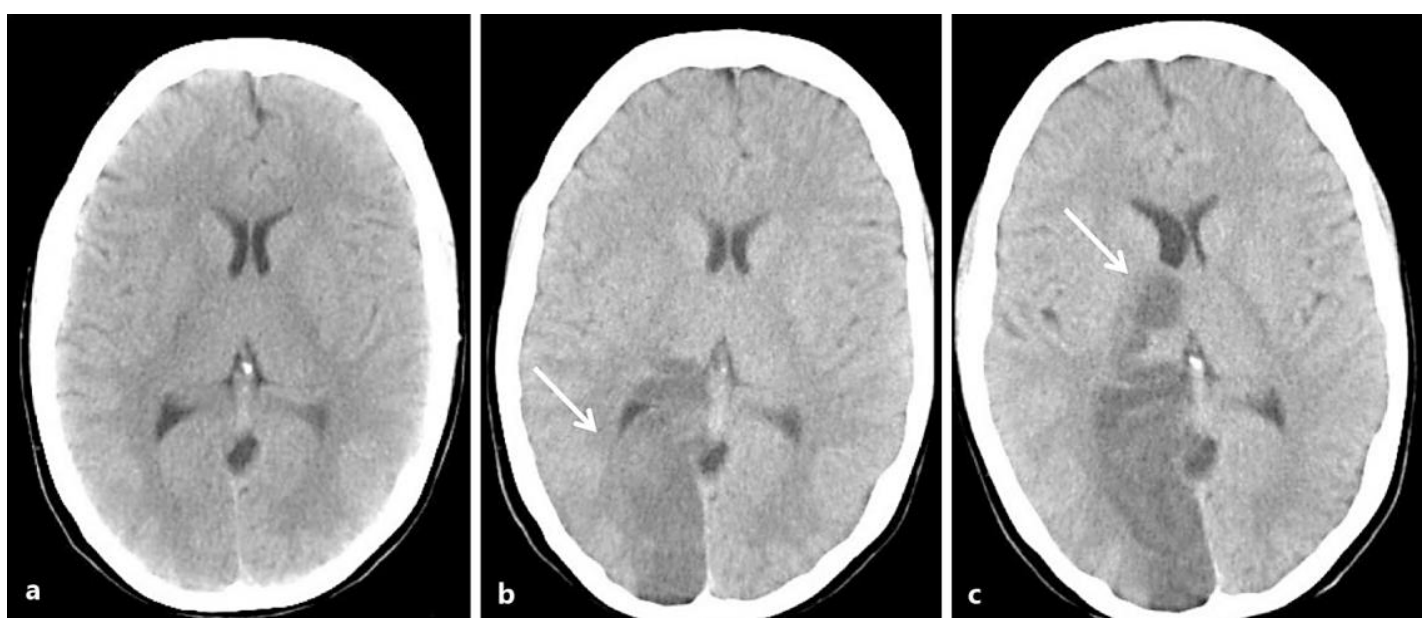

Fig. 1. Head CT scan performed in the emergency department (a), on the third day after the event (b), with a large hypodensity (arrow) on the territory of the right posterior cerebral artery, and 6 days after admission (c), showing enlargement of the ischemic lesion with involvement of the right thalamus (arrow). 


\section{Case Reports in Neurology}

\begin{tabular}{l|l}
\hline Case Rep Neurol 2016;8:145-150 \\
\hline $10.1159 / 000447296$ & $\begin{array}{l}\text { @ 2016 The Author(s). Published by S. Karger AG, Basel } \\
\text { www.karger.com/crn }\end{array}$ \\
\hline
\end{tabular}

Borbinha et al.: A Young Woman with Ischemic Stroke: Should We Pay More Attention to Varicella Zoster Infection?
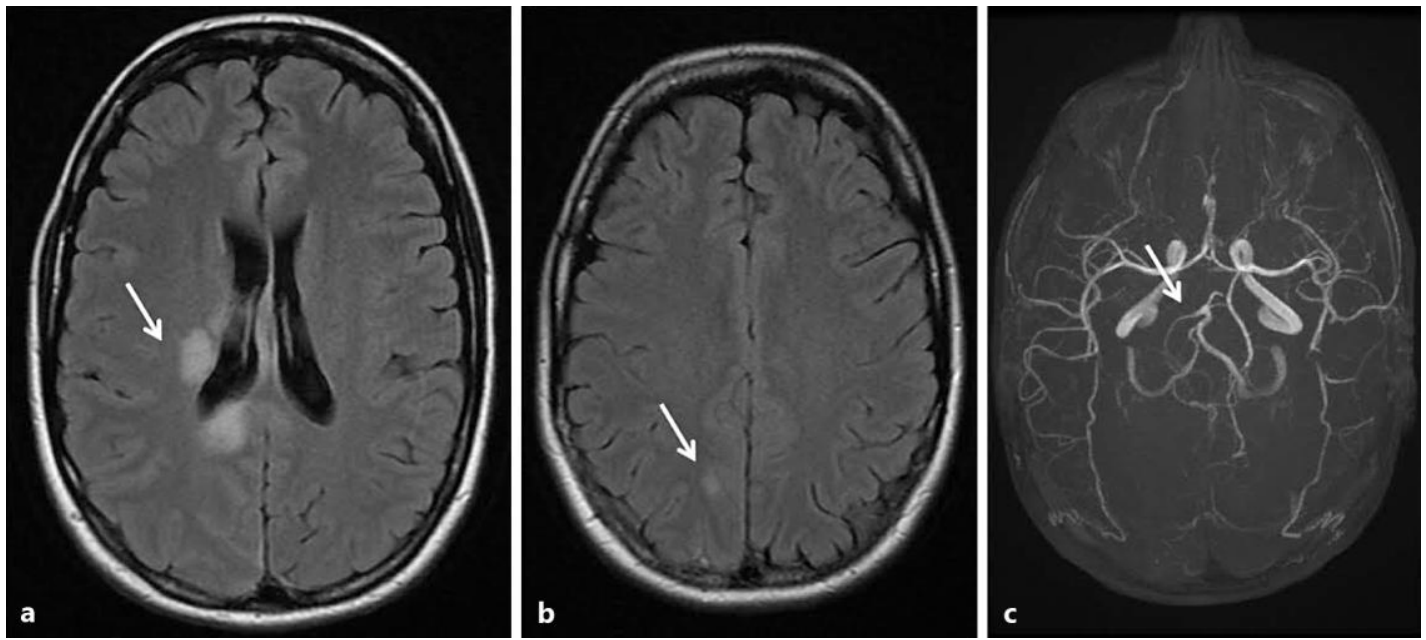

Fig. 2. Brain MRI performed on the seventh day after admission. T2 FLAIR images showed hyperintensity in the right periventricular white matter and posterior corpus callosum (a), and medial juxtacortical parietal region (b), indicative of ischemic lesions. MR angiography (TOF) showed occlusion of the right posterior cerebral artery $5 \mathrm{~mm}$ after its origin (c).
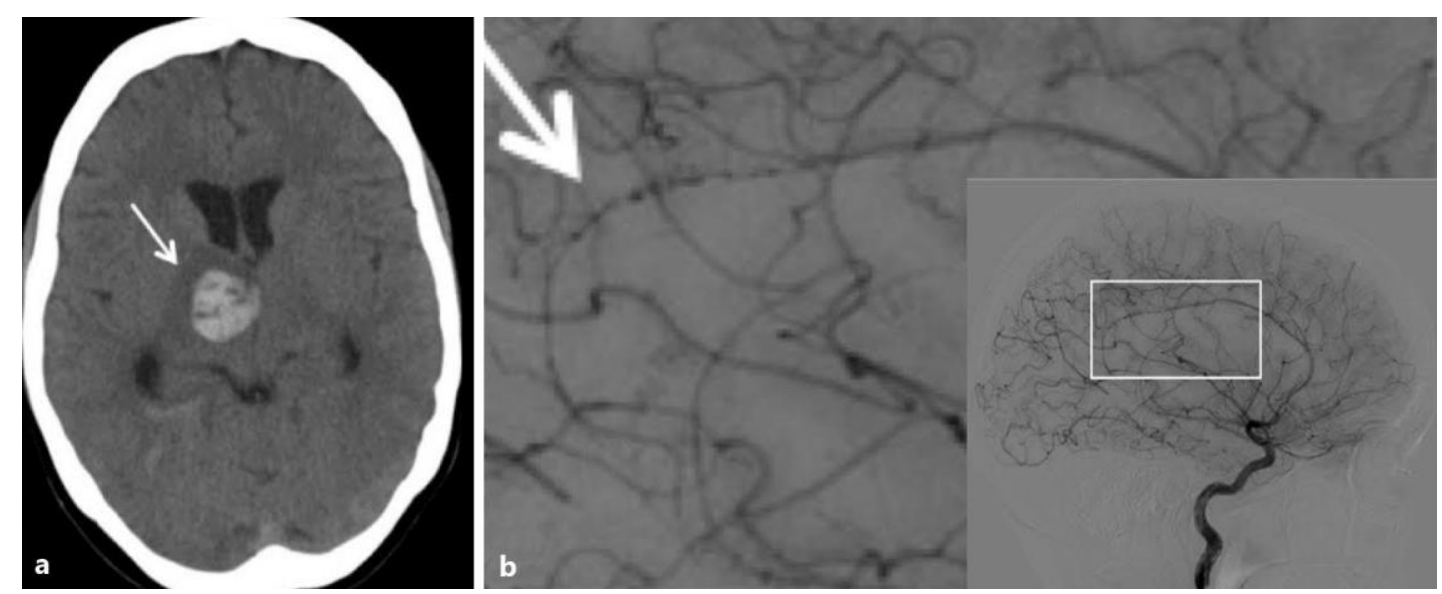

Fig. 3. a Head CT scan made 13 days after the ictal event showing hemorrhagic transformation involving the thalamus (arrow). b DSA of the left carotid artery showing an irregular lumen of the pericallosal artery with beading-like pattern appearance (arrow). 